\title{
Search for solar axions with the CAST experiment
}

\author{
Biljana Lakić $^{* 1}$, E. Arik ${ }^{17, j}$, S. Aune ${ }^{3}$, D. Autiero ${ }^{2, a}$, K. Barth ${ }^{2}$, A. Belov ${ }^{11}$,
} B. Beltrán ${ }^{6, b}$, S. Borghi' ${ }^{2, c}$, G. Bourlis ${ }^{18}$, F. S. Boydag ${ }^{17, j}$, H. Bräuninger ${ }^{5}$, J. M. Carmona ${ }^{6}$, S. Cebrián ${ }^{6}$, S. A. Cetin ${ }^{17}$, J. I. Collar ${ }^{7}$, T. Dafni ${ }^{6}$, M. Davenport ${ }^{2}$, L. Di Lella ${ }^{2, d}$, O. B. Dogan ${ }^{17, j}$, C. Eleftheriadis ${ }^{8}$, N. Elias ${ }^{2}$, G. Fanourakis ${ }^{9}$, E. Ferrer-Ribas ${ }^{3}$, H. Fischer ${ }^{10}$, P. Friedrich ${ }^{5}$, J. Franz $^{10}$, J. Galán ${ }^{6}$, T. Geralis ${ }^{9}$, I. Giomataris ${ }^{3}$, S. Gninenko ${ }^{11}$, H. Gómez ${ }^{6}$, R. Hartmann ${ }^{5}$, M. Hasinoff ${ }^{12}$, F. H. Heinsius ${ }^{10, e}$, I. Hikmet ${ }^{17, j}$, D. H. H. Hoffmann ${ }^{4}$, I. G. Irastorza ${ }^{6}$, J. Jacoby ${ }^{13}$, K. Jakovčić ${ }^{1}$, D. Kang ${ }^{10, f}$, K. Königsmann ${ }^{10}$, R. Kotthaus ${ }^{14}$, M. Krčmar ${ }^{1}$, K. Kousouris ${ }^{9, g}$, M. Kuster ${ }^{4,5}$, C. Lasseur ${ }^{2}$, A. Liolios ${ }^{8}$, A. Ljubičić ${ }^{1}$, G. Lutz ${ }^{14}$, G. Luzón ${ }^{6}$, D. Miller ${ }^{7}$, J. Morales ${ }^{6}$, T. Niinikoski ${ }^{2}$, A. Nordt ${ }^{4,5}$, A. Ortiz $^{6}$, T. Papaevangelou ${ }^{3}$, M. J. Pivovaroff ${ }^{16}$, A. Placci ${ }^{2}$, G. Raffelt ${ }^{14}$, H. Riege ${ }^{2,4}$, A. Rodríguez ${ }^{6}$, J. Ruz ${ }^{6}$, I. Savvidis ${ }^{8}$, Y. Semertzidis ${ }^{15, h}$, P. Serpico ${ }^{14, i}$, R. Soufli ${ }^{16}$, L. Stewart ${ }^{2}$, K. van Bibber ${ }^{16}$, J. Villar ${ }^{6}$, J. Vogel $^{10}$, L. Walckiers ${ }^{2}$, K. Zioutas ${ }^{15,2}$

${ }^{1}$ Rudjer Bošković Institute, Zagreb, Croatia

Email: Biljana. Lakic@irb.hr

${ }^{2}$ European Organization for Nuclear Research (CERN), Genève, Switzerland

${ }^{3}$ CEA, IRFU, Centre de Saclay, Gif-sur-Yvette, France

${ }^{4}$ Technische Universität Darmstadt, IKP, Darmstadt, Germany

${ }^{5}$ Max-Planck-Institut für extraterrestrische Physik, Garching, Germany

${ }^{6}$ Instituto de Física Nuclear y Altas Energías, Universidad de Zaragoza, Zaragoza, Spain

${ }^{7}$ Enrico Fermi Institute and KICP, University of Chicago, Chicago, IL, USA

${ }^{8}$ Aristotle University of Thessaloniki, Thessaloniki, Greece

${ }^{9}$ National Center for Scientific Research “Demokritos”, Athens, Greece

${ }^{10}$ Albert-Ludwigs-Universität Freiburg, Freiburg, Germany

${ }^{11}$ Institute for Nuclear Research (INR), Russian Academy of Sciences, Moscow, Russia

${ }^{12}$ Department of Physics and Astronomy, University of British Columbia, Vancouver, Canada

${ }^{13}$ Johann Wolfgang Goethe-Univ., Inst. für Angewandte Physik, Frankfurt am Main, Germany

${ }^{14}$ Max-Planck-Institut für Physik, München, Germany

${ }^{15}$ Physics Department, University of Patras, Patras, Greece

${ }^{16}$ Lawrence Livermore National Laboratory, Livermore, CA, USA

${ }^{17}$ Dogus University, Istanbul, Turkey

18 Present address: Hellenic Open University, Patras, Greece

The CERN Axion Solar Telescope (CAST) is searching for axions produced by the Primakoff process in the solar core. CAST is using a dipole magnet where axions could be converted back into X-rays. Phase I data analysis resulted in the most restrictive experimental limit on the axionphoton coupling constant for $m_{a} \lesssim 0.02 \mathrm{eV}$. In CAST Phase II, the sensitivity is extended to higher axion masses. Results of the analysis of 2005 and 2006 data, covering the range $m_{a} \lesssim 0.4 \mathrm{eV}$, will be presented. Currently CAST is searching for axions with masses up to $1.2 \mathrm{eV}$.

Identification of dark matter 2008

August 18-22, 2008

Stockholm, Sweden

${ }^{*}$ Speaker. 


\section{Introduction}

Axions are hypothetical particles arising in models which may explain the CP problem of strong interactions. The underlying Peccei-Quinn (PQ) mechanism [1] introduces a new global $U(1)$ chiral symmetry that is spontaneously broken at some large energy scale $f_{a}$. The consequence of the PQ mechanism is axion, the Nambu-Goldstone boson of $U(1)_{\mathrm{PQ}}$. Axion is practically stable neutral pseudoscalar and a candidate for the dark matter of the universe. The phenomenology is determined by the scale $f_{a}$. The axion mass can be expressed in the form $m_{a}=6 \mathrm{eV}\left(10^{6} \mathrm{GeV} / f_{a}\right)$. Most of the axion experimental searches are based on the axion interaction with two photons:

$$
\mathscr{L}_{a \gamma}=-\frac{1}{4} g_{a \gamma} F_{\mu v} \tilde{F}^{\mu v} a=g_{a \gamma} \mathbf{E} \cdot \mathbf{B} a,
$$

where $F$ is the electromagnetic field-strength tensor, $\tilde{F}$ its dual, $a$ the axion field, $\mathbf{E}$ the electric and B the magnetic field. The axion-photon coupling constant $g_{a \gamma}$ can be written in the form

$$
g_{a \gamma}=\frac{\alpha}{2 \pi f_{a}}\left(\frac{E}{N}-\frac{2(4+z+w)}{3(1+z+w)}\right)=\frac{\alpha}{2 \pi f_{a}}\left(\frac{E}{N}-1.92 \pm 0.08\right)
$$

where $z \equiv m_{u} / m_{d}$ and $w \equiv m_{u} / m_{s}$ are quark-mass ratios and $E / N$ is the model-dependent parameter. As a consequence of this interaction, axions could transform into photons and vice versa in external electric and magnetic fields. The CAST experiment is based on the axion helioscope technique [2] where a dipole magnet is oriented towards the Sun. Axions could be produced in the solar plasma by converting thermal photons in the Coulomb fields of nuclei and electrons - the Primakoff process, and back-converted into photons in a laboratory magnetic field. The expected solar axion flux at the Earth is $\Phi_{a}=3.75 \times 10^{11}\left(g_{a \gamma} /\left(10^{-10} \mathrm{GeV}^{-1}\right)\right)^{2} \mathrm{~cm}^{-2} \mathrm{~s}^{-1}$ with an approximate spectrum

$$
\frac{d \Phi_{a}}{d E_{a}}=6.02 \times 10^{10}\left(\frac{g_{a \gamma}}{10^{-10} \mathrm{GeV}^{-1}}\right)^{2} \frac{\left(E_{a} / \mathrm{keV}\right)^{2.481}}{e^{E_{a} / 1.205 \mathrm{keV}}} \mathrm{cm}^{-2} \mathrm{~s}^{-1} \mathrm{keV}^{-1}
$$

and the average energy $\left\langle E_{a}\right\rangle=4.2 \mathrm{keV}$. The expected number of photons (X-rays) reaching a detector is $N_{\gamma}=\int\left(d \Phi_{a} / d E_{a}\right) P_{a \rightarrow \gamma} S t d E_{a}$ where $P_{a \rightarrow \gamma}$ is the axion-photon conversion probability, $S$ the effective area and $t$ the measurement time. The axion-photon conversion probability in vacuum can be written as $P_{a \rightarrow \gamma}=\left(g_{a \gamma} B / q\right)^{2} \sin ^{2}(q L)$ where $L$ is the magnet length, $B$ the magnetic field and $q=m_{a}^{2} / 2 E_{a}$ the axion-photon momentum difference. For axions and photons to be in phase along the magnet length, the coherence condition $q L<\pi$ has to be satisfied. Therefore, the experimental sensitivity is restricted to a range of axion masses (for example, $m_{a} \lesssim 0.02 \mathrm{eV}$ for $L=10 \mathrm{~m}$ and $\left.E_{a}=4.2 \mathrm{keV}\right)$. In order to extend the sensitivity to higher axion masses, the conversion region has to be filled with a buffer gas which provides an effective photon mass $m_{\gamma}$. In that case, the conversion probability takes the form [3]

$$
P_{a \rightarrow \gamma}=\left(\frac{B g_{a \gamma}}{2}\right)^{2} \frac{1}{q^{2}+\Gamma^{2} / 4}\left(1+e^{-\Gamma L}-2 e^{-\Gamma L / 2} \cos (q L)\right)
$$

where $q=\left|m_{a}^{2}-m_{\gamma}^{2}\right| /\left(2 E_{a}\right)$ and $\Gamma$ is the inverse absorption length for photons in a gas. As a result, the coherence is restored for a narrow mass window around $m_{a}=m_{\gamma}$ (figure 1). 


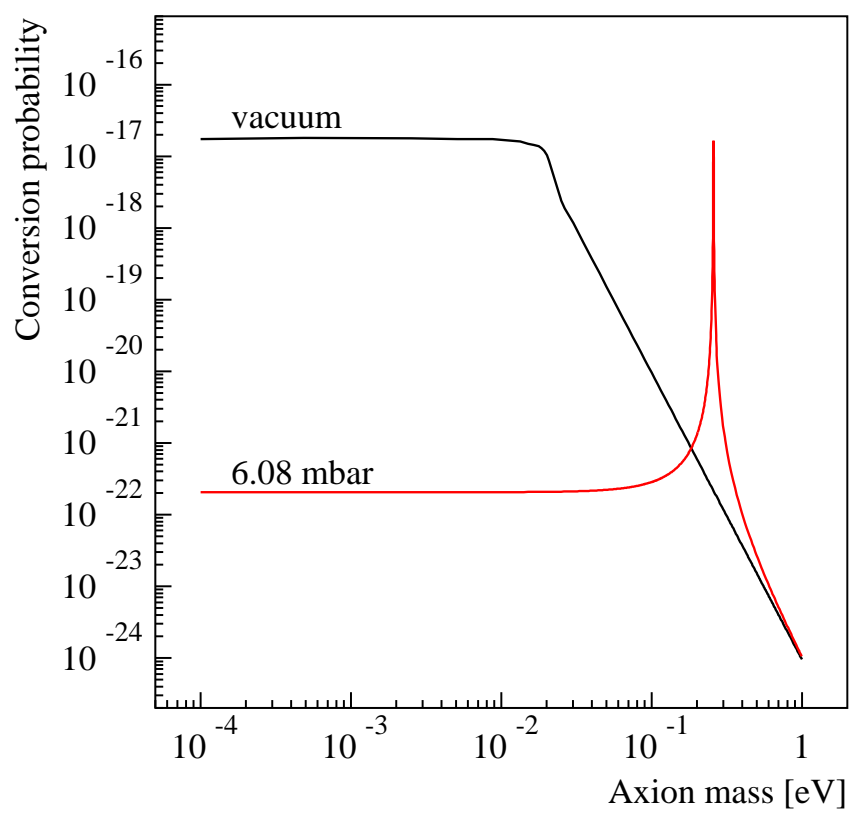

Figure 1: Axion-photon conversion probability versus axion mass. The black line corresponds to the case when vacuum is inside the conversion region and the red line to one particular helium pressure setting. The coupling constant of $1 \times 10^{-10} \mathrm{GeV}^{-1}$ is assumed.

The first implementation of the axion helioscope principle was performed in Brookhaven [4] and later a more sensitive search in Tokyo [5, 6, 7]. The most sensitive heliscope experiment CAST $[8,9]$ has been taking data since 2003 , both with vacuum and gas (first ${ }^{4} \mathrm{He}$ and later ${ }^{3} \mathrm{He}$ ) inside the conversion region.

\section{Experimental setup}

The external magnetic field in the CAST experiment is provided by a Large Hadron Collider (LHC) prototype dipole magnet [10] with the magnetic field $B=9.0 \mathrm{~T}$. Inside the magnet there are two paralell, straight pipes with the length $L=9.26 \mathrm{~m}$ and cross-sectional area $S=2 \times 14.5 \mathrm{~cm}^{2}$. The operating temperature is $1.8 \mathrm{~K}$ which is provided by a full cryogenic station. The magnet is mounted on the rotating platform with $\pm 40^{\circ}$ horizontal and $\pm 8^{\circ}$ vertical movement. As a result, the Sun can be observed for 1.5 hours at both sunrise and sunset during the whole year. At both ends of the magnet, different detectors are searching for photons coming from axion conversion inside the magnet when it is pointing to the Sun. The time the Sun is not reachable is used for background measurements. Periodical so-called GRID measurements show that CAST points to the Sun within the required precision. As an additional check, the Sun can be filmed twice per year using a camera placed on the magnet. The overall tracking precision is $\sim 0.01^{\circ}$.

For the data taking with ${ }^{4} \mathrm{He}$, a gas system was designed to operate in range $0-16.4$ mbar at $1.8 \mathrm{~K}$. The system provided a homogenous and stable density along the magnet bores, with 
adequate accuracy and reproducibility of density settings. At the ends of the bores, four X-ray windows were installed. The windows were designed to meet special requirements: high X-ray transmission (polypropylene $15 \mu \mathrm{m}$ ), resistance to sudden rise of pressure (strongback mesh) and minimum helium leakage.

Before 2007, CAST utilized the following X-ray detectors: a conventional Time Projection Chamber (TPC)[11], an unshielded Micromegas detector [12] and an X-ray mirror telescope in combination with a Charged Coupled Device (CCD) [13]. The X-ray focusing system and Micromegas were looking for sunrise axions, while the TPC was occupying both bores on the other end of the magnet looking for sunset axions. The X-ray telescope can focus the photons to a $\sim 9 \mathrm{~mm}^{2}$ spot on the $\mathrm{CCD}$, thus significantly improving the experimental sensitivity.

\section{Operation, results and prospects}

The operation of the CAST experiment has been forseen to proceed in several phases:

- Phase I: during 2003 and 2004 the experiment operated with vacuum inside the magnet bores, thus exploring the axion mass range up to $0.02 \mathrm{eV}$. Data analysis showed the absence of excess photons when the magnet was pointing to the Sun, and therefore set an upper limit on the axion-photon coupling of $g_{a \gamma}<8.8 \times 10^{-11} \mathrm{GeV}^{-1}$ at $95 \%$ C.L. [9]. This result is the best experimental limit for the range of axion masses up to $0.02 \mathrm{eV}$, also superseeding the astrophysical limit derived from energy-loss arguments on horizontal branch stars (figure 2).

- Phase II with ${ }^{4} \mathrm{He}$ : during 2005 and 2006 the magnet pipes were filled with ${ }^{4} \mathrm{He}$. The gas pressure was increased from 0 to 14 mbar in appropriate steps to cover equally the accessible mass range. With 160 different pressure settings, the range of axion masses up to $0.39 \mathrm{eV}$ was scanned. The resulting upper limit on the axion-photon coupling constant [14] is shown in figure 2. The measurement time at each pressure setting was only a few hours, resulting in small event numbers and therefore large statistical fluctuations of the line contour. For the first time in the helioscope type of experiments, the limit has enetred the QCD axion model band in the electronvolt range.

- Phase II with ${ }^{3} \mathrm{He}$ : In 2008, CAST started taking data with ${ }^{3} \mathrm{He}$ inside the magnet pipes. The data taking will continue until the end of 2010. The range of axion masses up to $\sim 1.2 \mathrm{eV}$ will be scanned. Prospects for the ${ }^{3} \mathrm{He}$ phase are shown in figure 2 .

Apart from the main line of research, CAST could also be sensitive to axions from M1 nuclear transition [15], Kaluza-Klein [16] and low energy axions.

\section{Upgrades for the ${ }^{3} \mathrm{He}$ phase}

During 2007, the CAST experiment made several upgrades in order to prepare for the more demanding ${ }^{3} \mathrm{He}$ part of phase II data taking. The most important upgrade was a design and installation of a sophisticated and complex ${ }^{3} \mathrm{He}$ gas system [17]. The system includes high accuracy in 


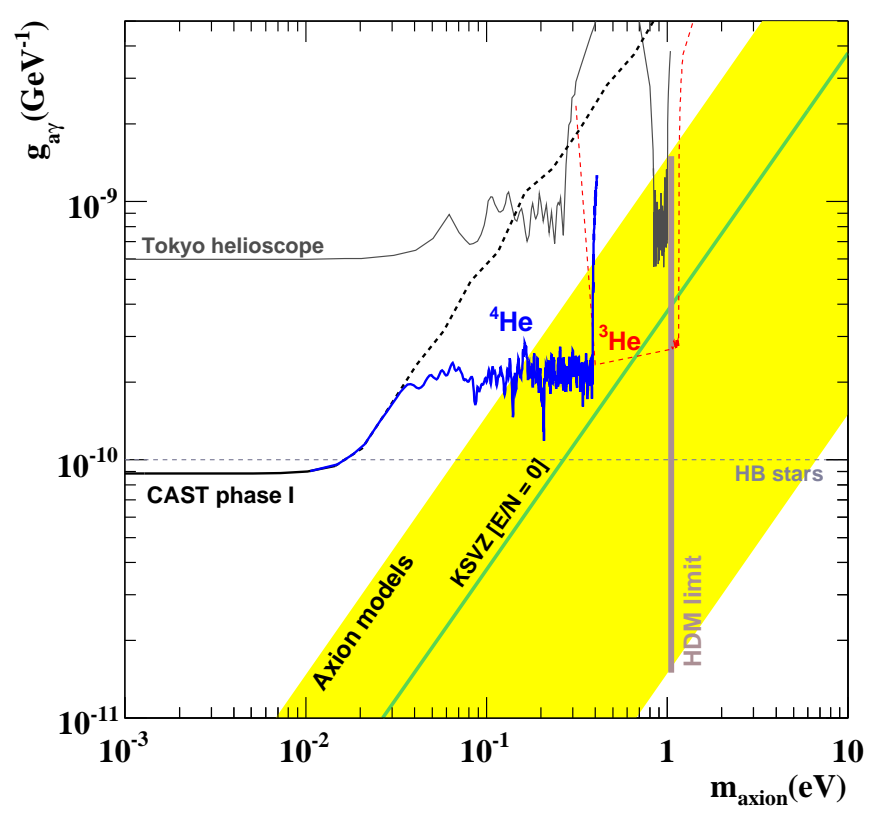

Figure 2: Exclusion limit on the axion-photon coupling constant versus axion mass. Combined result from the CAST phase I and ${ }^{4} \mathrm{He}$ part of phase II (blue line) is compared with results from the Tokyo experiment and horizontal branch starts limit. The HDM line refers to the hot dark matter limit [18]. The yellow band represents typical theoretical models while the red dashed line shows CAST prospects for the ${ }^{3} \mathrm{He}$ run.

measuring the gas quantity, flexible operation modes (stepping and ramping), absence of thermoacoustic oscillations and protection of cold, thin X-ray windows during a quench. It is a hermetic system in order to avoid the loss of ${ }^{3} \mathrm{He}$.

During this period, CAST detectors were upgraded as well: a new shielded bulk Micromegas replaced the unshielded one, while the TPC detector was replaced by two shielded Micromegas detectors (bulk and Microbulk). Upgraded detectors have a very low background level, therefore improving the experimental sensitivity in the ongoing ${ }^{3} \mathrm{He}$ part of phase II.

\footnotetext{
${ }^{a}$ Present address: Institute de Physique Nucléaire, Lyon, France

${ }^{b}$ Present address: Department of Physics, Queen's University, Kingston, Ontario, Canada

${ }^{c}$ Present address: Department of Physics and Astronomy, University of Glasgow, Glasgow, UK

${ }^{d}$ Present address: Scuola Normale Superiore, Pisa, Italy

e Present address: Ruhr-Universität Bochum, Bochum, Germany

${ }^{f}$ Present address: Institut für Experimentalle Kernphysik, Universität Karlsruhe, Karlsruhe, Germany

${ }^{g}$ Present address: Fermi National Accelerator Laboratory, Batavia, Illinois, USA

${ }^{h}$ Present address: Brookhaven National Laboratory, Upton, New York, USA

${ }^{i}$ Present address: European Organization for Nuclear Research (CERN), Genev̀e, Switzerland

${ }^{j}$ Deceased
} 


\section{Conclusions}

The result from the CAST phase I data is the best laboratory limit on the axion-photon coupling constant for $m_{a} \lesssim 0.02 \mathrm{eV}$. In the ${ }^{4} \mathrm{He}$ part of CAST phase II, the search has entered the QCD axion model band and set a mean upper limit of $2.17 \times 10^{-10} \mathrm{GeV}^{-1}$ in the range $0.02<m_{a}<0.26 \mathrm{eV}$. The ${ }^{3} \mathrm{He}$ part of phase II will explore the range up to $\sim 1.2 \mathrm{eV}$. CAST phase II explores the mass range where QCD axions would provide a hot dark matter component similar to neutrinos.

\section{Acknowledgments}

We acknowledge support from MSES (Croatia) under grant no. 098-0982887-2872 and partial support by the European Union under the ILIAS project, contract no. RII3-CT-2004-506222.

\section{References}

[1] R. D. Peccei and H. R. Quinn, Phys. Rev. Lett. 38, 1440 (1977); Phys. Rev. D16, 1791 (1977).

[2] P. Sikivie, Phys. Rev. Lett. 51, 1415 (1983) [Erratum-ibid. 52, 695 (1984)].

[3] K. van Bibber et al., Phys. Rev. D 39, 2089 (1989).

[4] D. M. Lazarus et al., Phys. Rev. Lett. 69, 2333 (1992).

[5] S. Moriyama et al., Phys. Lett. B 434, 147 (1998).

[6] Y. Inoue et al., Phys. Lett. B 536, 18 (2002).

[7] Y. Inoue et al., Phys. Lett. B 668, 93 (2008).

[8] K. Zioutas et al., Phys. Rev. Lett. 94, 121301 (2005).

[9] S. Andriamonje et al., JCAP 04, 10 (2007).

[10] K. Zioutas et al., Nucl. Instrum. Meth. A 425, 480 (1999).

[11] D. Autiero et al., New. J. Phys. 9, 171 (2007).

[12] P. Abbon et al., New. J. Phys. 9, 170 (2007).

[13] M. Kuster et al., New. J. Phys. 9, 169 (2007).

[14] E. Arik et al., submitted to JCAP [arXiv:0810.4482 [hep-ex]].

[15] K. Jakočić et al., Nucl. Instrum. Meth. A 580, 37 (2007).

[16] R. Horvat, M. Krčmar and B. Lakić, Phys. Rev. D 69, 125011 (2004).

[17] CAST Collaboration, SPSC-TDR-001, CERN-SPSC-2006-029.

[18] S. Hannestad, A. Mirizzi, G. G. Raffelt and Y. Y. Y. Wong, JCAP 0804, 019 (2008). 